\title{
Response to letter to the editor regarding the article "SGLT2 inhibitors and cardiovascular and renal outcomes: a meta-analysis and trial sequential analysis" Cardiac, renal protection with SGLT2 inhibitors is evident but not in every diabetic patient
}

\author{
Mahmoud Barbarawi $^{1}$. Owais Barbarawi ${ }^{2} \cdot$ Kai Chen $^{1}$ \\ Accepted: 12 April 2021 / Published online: 4 May 2021 \\ (c) The Author(s), under exclusive licence to Springer Science+Business Media, LLC, part of Springer Nature 2021
}

Qiu et al. conducted a meta-regression analysis on our newly published updated meta-analysis [1] to assess the benefit of the sodium-glucose cotransporter 2 (SGLT2) inhibitors in subgroups of diabetic patients with regard to comorbidities such as atherosclerotic cardiovascular disease (ASCVD), chronic kidney disease (CKD), or heart failure (HF). Their findings are in agreement with our conclusion that confirmed the efficacy of SGLT2 inhibitors in reducing the incidence of CKD progression and cardiovascular events including HF hospitalization, cardiovascular mortality, all-cause mortality, and major adverse cardiovascular events. Furthermore, they concluded the efficacy of SGLT2 in the diabetic patients independent of their status of ASCVD, CKD, or HF.

Meta-regression is an important extension to the metaanalysis through subgroup analysis that allows further investigation of the effect of categorical and continuous variables. However, precaution should be taken when investigating the effect of multiple variables simultaneously with limited number of studies such as 10 trials here and only study level data being accessible [2]. While the results of SGLT2 by analyzing each one of three variables (ASCVD, CKD, and HF) at a time seem to be consistently beneficial, a conclusion of overall efficacy in the diabetic patients with or without ASCVD, CKD, or HF may be misleading.
Our meta-analysis included 10 randomized trials that evaluated the benefit of SGLT2 inhibitors in patients with established cardiovascular disease, patients with multiple risk factors for cardiovascular disease, or patients with nephropathy as illustrated in Table 1 of our study. Qiu et al. provided the results that the effect of the SGLT2 inhibitors was consistent in diabetic patients with or without ASCVD, with or without CKD, and with or without HF. However, it must be emphasized that patients with diabetes who have none of the variables (ASCVD, CKD, and $\mathrm{HF}$ ) were not included in any of the published clinical trials [1] and may not have cardiovascular and renal benefit from SGLT2 inhibitors.

\section{References}

1. Barbarawi M, Al-Abdouh A, Barbarawi O et al (2021) SGLT2 inhibitors and cardiovascular and renal outcomes: a meta-analysis and trial sequential analysis. Heart Fail Rev. https://doi.org/10. 1007/s10741-021-10083-z

2. Thompson SG, Higgins JPT (2002) How should meta-regression analyses be undertaken and interpreted? Stat Med 21:1559-1573. https://doi.org/10.1002/sim.1187

Publisher's Note Springer Nature remains neutral with regard to jurisdictional claims in published maps and institutional affiliations.

Mahmoud Barbarawi

Mahmoud.albarbarawi@gmail.com; Barbawi@uchc.edu

1 Department of Cardiology, University of Connecticut, 263

Farmington Avenu, Farmington, CT, USA

2 Department of Internal Medicine, Mutah University, Al-Karak, Jordan 